\title{
Scattering of Incident P-Waves by a Semicylindrical Core-Shell Structure in an Elastically Constrained Half-Space at Nanoscale
}

\author{
R. Zhang (D, D. X. Lei, and Z. Y. Ou \\ School of Science, Lanzhou University of Technology, Lanzhou, Gansu 730050, China \\ Correspondence should be addressed to Z. Y. Ou; zhiyingou@163.com
}

Received 11 January 2020; Accepted 24 March 2020; Published 14 June 2020

Academic Editor: Pawel Packo

Copyright (c) 2020 R. Zhang et al. This is an open access article distributed under the Creative Commons Attribution License, which permits unrestricted use, distribution, and reproduction in any medium, provided the original work is properly cited.

Under the premise of the theory of surface elasticity, the scattering of plane compressional waves (P-waves) in the surface of a semicylindrical core-shell structure within the nanoscale elastic confinement half-space is studied by using the method of eigenfunction expansion. The generalized Y-L equation is used to give the nanoscale boundary conditions, and the dynamic stress concentration factor (DSCF) along the interface of core-shell structure induced by the plane elastic wave are derived and numerically evaluated. Under different incident wave frequencies, surface energy, and shear modulus, when the radius of the coreshell structure is reduced to the nanometer level, their influence on the DSCF is very significant. These have been confirmed by numerical calculations.

\section{Introduction}

The scattering of elastic waves by using a single scatterer with uniform curvature (e.g., cylindrical and spherical) embedded in an elastic matrix becomes an important topic in wave motion, due to the significant role of scattering in understanding various wave propagation phenomena in engineering materials and structures [1]. In particular, the scattering of an elastic wave by a semicylindrical structure is used as a simple model to investigate the wave motion properties of a tunnel or sedimentary valley. Twerksy [2] first studied the multiple scattering of waves by multiple spheres and cylinders in a fluid and laid the foundation for multiple scattering, such as multiple scattering of dense objects $[3,4]$, grating scattering [5], and propagation of $\mathrm{P}$-waves in fiberreinforced composites [6-8]. Pao and Mow [9] and Mow and Mente [10] discussed the dynamic stress concentration around cylindrical inclusions and used the wave function expansion method to comprehensively study the scattering of elastic waves by cylindrical inclusions and sphere inclusions. Roumeliotis and Fikioris [11] analyzed the cutoff wavenumbers and the field of surface wave modes of a circular cylindrical conductor eccentrically coated by a dielectric. Trifunac [12] used the separation variable method to obtain the solution of the scattered waves of the plane SHwaves on the semicircular sedimentary valley in the halfspace, the closed-form solution of the problem shows that the surface topography can influence on incident waves only when the wavelengths of incident motion are short compared to the radius of a canyon. Weber et al. [13] derived the scattering behavior of a single multilayered inclusion in a homogeneous isotropic matrix under the influence of nonplane elastic SH-waves. Liang et al. [14] studied the analytic solution of scattering of incident plane P-waves by circular-shaped layered sedimentary valleys, and the solution was utilized to analyze the effects of alluvial sequence and their relative stiffness on the scattering of incident waves. Bo et al. [15] analyzed three kinds of resonant modes of a single layered circular elastic cylinder embedded in the elastic medium. Li et al. [16] gave an analytical solution for the scattering of $\mathrm{P}$-waves by cylindrical inclusions in a halfspace at the macroscopic scale, and it was illustrated that there was great difference of the diffraction characteristics between the hard inclusion and soft inclusion, and the displacement response depended strongly on the incident angle and frequency. Shindo and Niwa [17] dealt with the scattering of antiplane shear waves in a metal matrix composite reinforced by fibers with interracial layers. Lee 
and Liu [18] studied the two-dimensional scattering and diffraction of $\mathrm{P}$-waves and $\mathrm{SV}$-waves around a semicircular canyon in an elastic half space by using the analytical solution of the stress-free wave function. Xu et al. [19] and Cao et al. [20] used the complex variable function method to study the SH-waves scattering problem of various models in the elastic half-space.

However, in the aforementioned studies, the effect of the interface stress was not taken into account. Wang [21, 22] studied the scattering of cylindrical pores by nanoscale plane compression waves under the interface effect and considered the diffraction problem of P-waves with two circular holes and inclusions in a semi-infinite plane under the surface/ interface effect, and the results show that surface/interface had a significant effect on the diffractions of elastic waves as the radius of the inclusion shrinks to nanoscale. Ou and Lee [23] studied the scattering of planar elastic waves by layer fibers with surface/interface effects at the nanoscale, using the displacement potential method. Ru et al. [24] researched the diffraction of elastic waves and the stress concentration near cylindrical nanoinclusions with surface/interface effects, indicating that the influence of the surface/interface on the elastic wave diffraction becomes remarkable when the radius of the inclusion or cavity is reduced to the nanometer level.

In addition, nanocomposites with a core-shell structure have become hotspots in composite nanomaterials and other fields in recent years. The core-shell nanocomposites (CSNC) generally consists of a central core and an outer shell layer. The inner core and outer shell in CSNC are connected to each other through physical and chemical interactions, and the core and the shell are different substances [25]. The core-shell composite nanoparticles formed by the combination of two or more materials at the nanoscale are novel composite nanostructures. This structure can produce many new properties such as photoelectric conversion, nonlinear optics, electromagnetic conversion, solar cells, and highdensity information storage [26-29]. Therefore, nanocomposites with a core-shell structure have wider research prospects which have expanded into the interdisciplinary fields of chemistry, physics, biology, materials, and other disciplines, in biomedicine, health care products, cosmetics, environmental protection and other fields, having broad application potential [30-32]. However, in the process of material preparation, nanometer core-shell structures of different shapes are often formed like cylindrical, elliptic cylindrical, spherical, and ellipsoidal. These geometric shapes are bound to bring certain difficulties to study the elastic wave scattering of nanometer materials $[33,34]$. In summary, the study on the scattering of elastic waves by a core-shell structure with surface effect has theoretical guidance for the design and processing of nanomaterials and nanometer components. However, the existing literature shows that research on the scattering of elastic waves by macroscopic heterogeneous media and nanoinclusions and holes with surface/interface effects has achieved certain results, but there is a lack of research on elastic waves by surface/interface effects core-shell structures. In this paper, the scattering of plane compressional waves by a semicylindrical core-shell structure is studied with consideration of surface effects.

In Section 2, the semicylindrical core-shell structure model used in this study is described. The boundary conditions and governing equations for the elastic wave diffraction problem of the semicylindrical core-shell structure are given. The solution of the elastic field caused by the incident P-waves on the surface of the semicylindrical coreshell structure is obtained. In Section 3, the effects of lowfrequency and high-frequency incident P-waves and different shear modulus ratios on stress concentration are discussed. Section 4 gives conclusions.

\section{Statement of the Problem}

Based on the theory of surface elasticity, we consider elastic waves scattered by a semicylindrical core-shell structure. As shown in Figure 1, the superscripts 1 and 2 indicate the quantities associated with the matrix and the shell, respectively. For convenience, the Cartesian coordinate system $(x, y, z)$ with origin $o$ at the center of the semicylindrical core-shell structure and the corresponding cylindrical coordinate system $(r, \theta, z)$ are adopted, where the $z$-axis is along the central line of the semicylindrical core-shell structure. The center of the inner and outer semicircles of the core-shell structure are at the point $o$, the inner diameter is $b$, and the outer diameter is $a$. The curve is $r=a$ recorded as $L_{1}$, and the curve is $r=b$ recorded as $L_{2}$. The material properties of the half space are given by the Lame constant $\lambda_{1}, \mu_{1}$, and the mass density $\rho_{1}$, respectively, and the material properties of the shell are given by the Lame constant $\lambda_{2}, \mu_{2}$, and the mass density $\rho_{2}$, respectively. It is assumed that the P-waves propagate in the medium at the incident angle $\alpha$ and interact with the semispace straight boundary and the semicircular core-shell structure, thereby generating reflected $\mathrm{P}$-waves and SV-waves on the straight boundary and scattered $\mathrm{P}$-waves and $\mathrm{SV}$-waves on $L_{1}$ and standing waves (including refracted waves on $L_{1}$ and scattered waves on $L_{2}$ ) in the shell.

In bulk, the classical theory of elasticity is still applicable, but the presence of interface/surface stresses results in nonclassical boundary conditions. The surface stress $\sigma_{\alpha \beta}^{s}$ is connected with the surface/interface energy density $\Gamma$ by

$$
\sigma_{\alpha \beta}^{s}=\Gamma \delta_{\alpha \beta}+\frac{\partial \Gamma}{\partial \varepsilon_{\alpha \beta}},
$$

where $\delta_{\alpha \beta}$ is the Kronecker delta and $\varepsilon_{\alpha \beta}$ is the component of the second order tensor of surface strains.

Usually, a crystal surface is anisotropic. It would be quite difficult to obtain analytical formulas for the difference between the surface and volume properties of materials. Therefore, isotropic surfaces are usually simplified. This simplified method can not only obtain the main characteristics of the problem under consideration but also obtain some analytical results of isotropic surface stress $\sigma_{\alpha \beta}^{s}$ given by

$$
\sigma_{\alpha \beta}^{s}=\tau^{0} \delta_{\alpha \beta}+2\left(\mu^{s}-\tau^{0}\right) \delta_{\alpha \gamma} \delta_{\gamma \beta}+\left(\lambda^{s}+\tau^{0}\right) \varepsilon_{\gamma \gamma} \delta_{\alpha \beta},
$$

where $\mu^{s}$ and $\lambda^{s}$ are the surface Lame coefficients which characterize surface properties of the material. We notice 


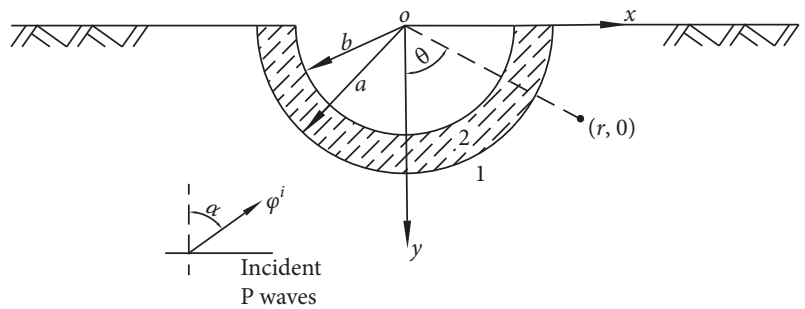

Figure 1: Semicylindrical core-shell structure.

that the residual surface tension $\tau^{0}$ will induce an additional static deformation field, and this will be neglected in our dynamic analysis.

Assume that the interface is attached to the bulk material without slipping and then obtains the equilibrium conditions on the interface

$$
\begin{aligned}
t_{\alpha}+\sigma_{\alpha \beta}^{s} & =0, \\
\left\langle\sigma_{i j}\right\rangle n_{i} n_{j} & =\sigma_{\alpha \beta}^{s} \kappa_{\alpha \beta},
\end{aligned}
$$

where $n_{i}$ represent the normal vector of the interface direction toward the outside of the shell, $t_{\alpha}$ is the tangential component of the traction $t_{i}=\left\langle\sigma_{i j}\right\rangle n_{j}$ in the $x_{\alpha}$ direction, $\left\langle\sigma_{i j}\right\rangle$ is the jump in the component of the bulk stress tensor across the interface, and $\kappa_{\alpha \beta}$ is the curvature of the interface. In the dynamic problem, the interface inertial forces are ignored in equation (3).

On the whole, the classical elasticity theory holds, and the equilibrium equations and the isotropic constitutive relations are

$$
\begin{aligned}
\sigma_{i j, j} & =\rho \frac{\partial^{2} u_{i}}{\partial t^{2}} \\
\sigma_{i j} & =2 \mu\left(\varepsilon_{i j}+\frac{\nu}{1-2 \nu} \varepsilon_{k k} \delta_{i j}\right),
\end{aligned}
$$

where $\rho$ is the mass density of the material, $t$ is the time, $\mu$ is the shear modulus, $\nu$ is Poisson's ratio, $\sigma_{i j}$ is the components of the stress tensor, $\varepsilon_{i j}$ is the strain tensor in the bulk material, and $u_{i}$ is the component of displacement. The strain tensor is related to the displacement vector $\mathbf{u}$ by

$$
\varepsilon_{i j}=\frac{1}{2}\left(u_{i, j}+u_{j, i}\right)
$$

For the current plane strain problem, the interfacial strain components can be obtained by using the bulk stress

$$
\varepsilon_{\theta \theta}^{s}=\frac{1}{2 \mu}\left[(1-\nu) \sigma_{\theta \theta}-\nu \sigma_{r r}\right]
$$

and the interface stress $\sigma_{\theta \theta}^{s}$ can be obtained from equation (5).

$$
\sigma_{\theta \theta}^{s}=\left(2 \mu^{s}+\lambda^{s}\right) \varepsilon_{\theta \theta}^{s}
$$

and the boundary conditions satisfied on the straight boundary are

$$
\begin{aligned}
& \left.\sigma_{r \theta}\right|_{\theta=0, \pi}=0, \quad r>b, \\
& \left.\sigma_{\theta \theta}\right|_{\theta=0, \pi}=0, \quad r>b,
\end{aligned}
$$

at interface $L_{1}(r=a)$, and the continuity of the displacement needs to be

$$
\begin{gathered}
u_{r 1}=u_{r 2}, \\
u_{\theta 1}=u_{\theta 2}, \\
r=a .
\end{gathered}
$$

The equilibrium equations with interface effect are

$$
\begin{aligned}
\sigma_{r r 1}-\sigma_{r r 2} & =\frac{\sigma_{\theta \theta}^{s}}{a}, \\
\sigma_{r \theta 1}-\sigma_{r \theta 2} & =-\frac{1}{a} \frac{\partial \sigma_{\theta \theta}^{s}}{\partial \theta}, \\
r & =a, \\
\sigma_{r r 2} & =-\frac{\sigma_{\theta \theta}^{s}}{b}, \\
\sigma_{r \theta 2} & =\frac{1}{b} \frac{\partial \sigma_{\theta \theta}^{s}}{\partial \theta}, \\
r & =b,
\end{aligned}
$$

and substituting equation (7) into equation (8) and then equations (11) and (12), we get

$$
\begin{aligned}
\sigma_{r r 1}-\sigma_{r r 2} & =s_{2}\left[\left(1-\nu_{1}\right) \sigma_{\theta \theta 1}-\nu_{1} \sigma_{r r 1}\right], \quad r=a, \\
\sigma_{r \theta 1}-\sigma_{r \theta 2} & =-s_{2}\left[\left(1-\nu_{1}\right) \frac{\partial \sigma_{\theta \theta 1}}{\partial \theta}-v_{1} \frac{\partial \sigma_{r r 1}}{\partial \theta}\right], \quad r=a, \\
\sigma_{r r 2} & =-s_{1}\left[\left(1-\nu_{2}\right) \sigma_{\theta \theta 2}-v_{2} \sigma_{r r 2}\right], \quad r=b, \\
\sigma_{r \theta 2} & =s_{1}\left[\left(1-v_{2}\right) \frac{\partial \sigma_{\theta \theta 2}}{\partial \theta}-v_{2} \frac{\partial \sigma_{r r 2}}{\partial \theta}\right], \quad r=b,
\end{aligned}
$$

where

$$
\begin{aligned}
& s_{1}=\frac{2 \mu^{s}+\lambda^{s}}{2 b \mu_{2}}=\eta s_{2}, \\
& s_{2}=\frac{2 \mu^{s}+\lambda^{s}}{2 a \mu_{2}}, \\
& \eta=\frac{a}{b} .
\end{aligned}
$$

Equation (17) show that for macroscopic structures with large $a$ and $b$ values, $s_{1}, s_{2} \ll 1$, the influence of the interface can be ignored [23]. However, when the radius of the core-shell structure shrinks to the nanometer level, $s_{1}$ and $s_{2}$ become apparent, and the influence of the interface on the $\mathrm{P}$-waves scattering should be considered.

In the cylindrical coordinate system, the relative displacement represented by the compressive potential 
function $\phi$ and the shear potential function $\psi$ can be abbreviated as

$$
\begin{aligned}
& u_{r}=\frac{\partial \phi}{\partial r}+\frac{1}{r} \frac{\partial \psi}{\partial \theta}, \\
& u_{\theta}=\frac{1}{r} \frac{\partial \phi}{\partial \theta}-\frac{\partial \psi}{\partial r}, \\
& u_{z}=0,
\end{aligned}
$$

Therefore, the displacement in the matrix and the shell layer can be determined by equations (18a)-(18c), respectively.

The related stresses can be expressed by the compression potential function and the shear potential function as

$$
\sigma_{r r}=\lambda\left[\frac{\partial^{2} \phi}{\partial r^{2}}+\frac{1}{r} \frac{\partial \phi}{\partial r}+\frac{1}{r^{2}} \frac{\partial^{2} \phi}{\partial \theta^{2}}\right]+2 \mu\left[\frac{\partial^{2} \phi}{\partial r^{2}}-\frac{1}{r} \frac{\partial \psi}{\partial \theta}+\frac{1}{r} \frac{\partial^{2} \phi}{\partial r \partial \theta}\right],
$$

$$
\begin{aligned}
\sigma_{\theta \theta}= & \lambda\left[\frac{\partial^{2} \phi}{\partial r^{2}}+\frac{1}{r} \frac{\partial \phi}{\partial r}+\frac{1}{r^{2}} \frac{\partial^{2} \phi}{\partial \theta^{2}}\right]+2 \mu\left[\frac{1}{r^{2}} \frac{\partial^{2} \phi}{\partial \theta^{2}}+\frac{1}{r} \frac{\partial \phi}{\partial r}-\frac{1}{r} \frac{\partial^{2} \phi}{\partial r \partial \theta}\right. \\
& \left.+\frac{1}{r^{2}} \frac{\partial \psi}{\partial \theta}\right]
\end{aligned}
$$$$
\sigma_{r \theta}=\frac{\mu}{r}\left[2 \frac{\partial^{2} \phi}{\partial r \partial \theta}-\frac{2}{r} \frac{\partial \phi}{\partial \theta}+\frac{1}{r} \frac{\partial^{2} \psi}{\partial \theta^{2}}-r \frac{\partial^{2} \psi}{\partial r^{2}}+\frac{\partial \psi}{\partial r}\right],
$$

Therefore, the stresses in the matrix and the shell layer can be determined by equtaions (19a)-(19c), respectively.

2.1. Wave Equation on Straight Boundaries. Assume a simple harmonic plane P-wave propagating in a direction at an angle of $\alpha$ degrees to the vertical direction. The following displacement potential function can be used to represent the incident $\mathrm{P}$-waves:

$$
\phi^{(i)}(x, y)=\exp \left[i \alpha_{1}(x \sin \alpha-y \cos \alpha)-i \omega t\right],
$$

where $i^{2}=-1, t$ is the time, $\alpha_{1}=\omega / c_{p 1}$ is the compression wave number of the $\mathrm{P}$-waves, and $c_{p 1}=\sqrt{\left(\lambda_{1}+2 \mu_{1}\right) / \rho_{1}}$ is the compression waves velocity in the matrix material.

When there is no semicylindrical core-shell structure, the reflected waves on the straight boundary are

$$
\begin{aligned}
& \phi^{(r)}(x, y)=K_{1} \exp \left[i \alpha_{1}(x \sin \alpha+y \cos \alpha)-i \omega t\right], \\
& \psi^{(r)}(x, y)=K_{2} \exp \left[i \beta_{1}(x \sin \beta+y \cos \beta)-i \omega t\right],
\end{aligned}
$$

where $\beta_{1}=\omega / c_{s 1}$ is the wave number of the SV-waves, $c_{s 1}=$ $\sqrt{\mu_{1} / \rho_{1}}$ is the shear waves velocity in the matrix material, and the time factor $e^{-i \omega t}$ is negligible [23]. $K_{1}$ and $K_{2}$ are the reflection coefficients of the reflected $\mathrm{P}$-waves and the reflected SV-waves, respectively.

$$
\begin{aligned}
& K_{1}=\frac{\sin 2 \alpha \sin 2 \beta-k^{2} \cos ^{2} 2 \beta}{\sin 2 \alpha \sin 2 \beta+k^{2} \cos ^{2} 2 \beta}, \\
& K_{2}=\frac{-\sin 2 \alpha \sin 2 \beta}{\sin 2 \alpha \sin 2 \beta+k^{2} \cos ^{2} 2 \beta},
\end{aligned}
$$

where

$$
k^{2}=\frac{c_{p 1}^{2}}{c_{s 1}^{2}}
$$

Converting the incident waves and the reflected waves on the straight boundary into the polar coordinate system, we get

$$
\begin{aligned}
\phi^{(i)}(r, \theta) & =\exp \left[-i \alpha_{1} r \cos (\alpha+\theta)\right], \\
\phi^{(r)}(r, \theta) & =K_{1} \exp \left[i \alpha_{1} r \cos (\alpha+\theta)\right], \\
\psi^{(r)}(x, y) & =K_{2} \exp \left[i \beta_{1} r \cos (\beta+\theta)\right] .
\end{aligned}
$$

In the cylindrical coordinate system, the displacement potential functions of the incident and reflected waves in equations (24)-(26) can be rewritten with the Bessel function as

$$
\begin{aligned}
& \phi^{f f}(r, \theta)=\phi^{(i)+(r)}(r, \theta)=\sum_{n=0}^{\infty} J_{n}\left(\alpha_{1} r\right)\left(a_{n} \cos n \theta+b_{n} \sin n \theta\right), \\
& \psi^{f f}(r, \theta)=\psi^{(r)}(r, \theta)=\sum_{n=0}^{\infty} J_{n}\left(\beta_{1} r\right)\left(c_{n} \cos n \theta+d_{n} \sin n \theta\right),
\end{aligned}
$$

where

$$
\begin{aligned}
& a_{n}=\varepsilon_{n} i^{n}\left(K_{1}+(-1)^{n}\right) \cos n \alpha, \\
& c_{n}=K_{2} \varepsilon_{n} i^{n} \cos n \beta, \\
& b_{n}=\varepsilon_{n} i^{n}\left(K_{1}+(-1)^{n+1}\right) \sin n \alpha, \\
& d_{n}=K_{2} \varepsilon_{n} i^{n} \sin n \beta,
\end{aligned}
$$

where $\varepsilon_{n}$ is the Neumann factor (when $n=0, \varepsilon_{n}=1$; when $\left.n>0, \varepsilon_{n}=2\right)$ and $J_{n}(\cdot)$ is the first $n$-order Bessel function in the cylindrical coordinate system. The P-waves $\phi^{f f}$ and SVwaves $\psi^{f f}$ of the free-field together satisfy the zero-stress boundary conditions on the straight boundary.

2.2. Wave Equation on the Core-shell Structure. The general form of the scattered wave function is

$$
\begin{aligned}
\phi(r, \theta) & =\sum_{n=0}^{\infty} A_{n} H_{n}^{(1)} \sin n \theta, \\
\operatorname{or} \phi(r, \theta) & =\sum_{n=0}^{\infty} B_{n} H_{n}^{(1)} \cos n \theta, \\
\operatorname{or} \phi(r, \theta) & =\sum_{n=0}^{\infty} H_{n}^{(1)}\left(A_{n} \sin n \theta+B_{n} \cos n \theta\right) .
\end{aligned}
$$


The scattered waves here are either a cosine function, a sine function, or both. In the full-space problem, when $0 \leq \theta \leq 2 \pi$, usually the cosine and sine terms are included in the solution because they together form a complete set of orthogonal functions. But, in the half-space problem, when
$0 \leq \theta \leq \pi$, only the sine function or only the cosine function is orthogonal. As pointed out by Lee and Liu [18], a given sine function can be represented by a cosine function in the halfspace range (where $m=1,2,3, \ldots$ ).

$$
\sin m \theta=\sum_{\substack{n=0 \\ n+m=\text { odd }}}^{\infty} \frac{\varepsilon_{n}}{\pi} s_{m n} \cos n \theta= \begin{cases}-\sum_{\substack{n=0 \\ n+m=\text { odd }}}^{\infty} \frac{\varepsilon_{n}}{\pi} \frac{2 m}{m^{2}+n^{2}} \cos n \theta, & -\pi \leq 0 \leq 0, \\ \sum_{\substack{n=0 \\ n+m=\text { odd }}}^{\infty} \frac{\varepsilon_{n}}{\pi} \frac{2 m}{m^{2}+n^{2}} \cos n \theta, & 0 \leq 0 \leq \pi,\end{cases}
$$

where

$$
\begin{aligned}
s_{m n} & =\int_{0}^{\pi} \sin m \theta \cos n \theta \mathrm{d} \theta= \begin{cases}\frac{2 m}{m^{2}-n^{2}}, & n+m=\text { odd, } \\
0, & n+m=\text { even, }\end{cases} \\
\varepsilon_{n} & = \begin{cases}1, & n=0, \\
2, & n \geq 1 .\end{cases}
\end{aligned}
$$

Therefore, a completely independent solution in halfspace should contain only cosine functions or only sine functions, not both.

When the incident waves strike the semicylindrical coreshell structure, the P-waves are scattered back from the interface $L_{1}$, and the corresponding displacement potential of the scattered waves are given by

$$
\begin{aligned}
& \phi_{1}^{(s)}(r, \theta)=\sum_{n=1}^{\infty} A_{1 n} H_{n}^{(1)}\left(\alpha_{1} r\right) \sin n \theta, \\
& \psi_{1}^{(s)}(r, \theta)=\sum_{n=1}^{\infty} C_{1 n} H_{n}^{(1)}\left(\beta_{1} r\right) \sin n \theta,
\end{aligned}
$$

where $A_{1 n}$ and $C_{1 n}$ are the coefficients to be determined, and note that the summation will be changed from $n=1$ to $n=\infty$ because $A_{10}=0, C_{10}=0$.

Using equations (30) and (31), equations (32a) and (32b) can be expressed as a cosine function:

$$
\begin{aligned}
\phi_{1}^{(s)}(r, \theta)= & \sum_{m=1}^{\infty} H_{m}^{(1)}\left(\alpha_{1} r\right) A_{1 m} \sin m \theta=\sum_{m=1}^{\infty} H_{m}^{(1)}\left(\alpha_{1} r\right) A_{1 m} \\
& \cdot \sum_{\substack{n=0 \\
n+m=\text { odd }}}^{\infty} \frac{\varepsilon_{n}}{\pi} s_{m n} \cos n \theta,
\end{aligned}
$$

$$
\begin{aligned}
\psi_{1}^{(s)}(r, \theta)= & \sum_{m=1}^{\infty} H_{m}^{(1)}\left(\beta_{1} r\right) C_{1 m} \sin m \theta=\sum_{m=1}^{\infty} H_{m}^{(1)}\left(\beta_{1} r\right) C_{1 m} \\
& \cdot \sum_{\substack{n=0 \\
n+m=o d d}}^{\infty} \frac{\varepsilon_{n}}{\pi} s_{m n} \cos n \theta .
\end{aligned}
$$

The next step is to test how these wave functions satisfy the zero-stress boundary condition of the straight boundary.

2.2.1. Zero Normal Surface Stress in the Straight Boundary. Formula for zero normal surface stress in the straight boundary is

$$
\left.\sigma_{y y}\right|_{y=0}=\left.\sigma_{\theta \theta}\right|_{\theta=0, \pi}=0 .
$$

Using equations (32a)-(33b), the longitudinal wave potential function is represented by a sine series, and the transverse wave potential function is represented by a cosine series

$$
\begin{aligned}
& \phi_{1}^{(s)}(r, \theta)=\sum_{n=1}^{\infty} A_{1 n} H_{n}^{(1)}\left(\alpha_{1} r\right) \sin n \theta, \\
& \psi_{1}^{(s)}(r, \theta)=\sum_{n=0}^{\infty} \sum_{\substack{m=1 \\
n+m=\text { odd }}}^{\infty} C_{1 m} H_{m}^{(1)}\left(\beta_{1} r\right) \frac{\varepsilon_{n}}{\pi} s_{m n} \cos n \theta,
\end{aligned}
$$

and then the normal stress $\sigma_{\theta \theta}$ has only the term about $\sin n \theta$, so at $\theta=0$ and $\theta=\pi$, and $\sigma_{\theta \theta}=0$ satisfies the stressfree condition on the straight boundary.

2.2.2. Zero Shear Stress on the Straight Boundary. Formula for zero shear stress in the straight boundary is

$$
\left.\sigma_{y x}\right|_{y=0}=\left.\sigma_{r \theta}\right|_{\theta=0, \pi}=0 .
$$


Using equations (32a)-(33b), the longitudinal wave potential function is represented by a cosine series, and the transverse wave potential function is represented by a sine series

$$
\begin{aligned}
\phi_{1}^{(s)}(r, \theta) & =\sum_{n=o}^{\infty} \sum_{\substack{m=1 \\
n+m=\text { odd }}}^{\infty} A_{1 m} H_{m}^{(1)}\left(\alpha_{1} r\right) \frac{\varepsilon_{n}}{\pi} s_{m n} \cos n \theta, \\
\psi_{1}^{(s)}(r, \theta) & =\sum_{n=1}^{\infty} C_{1 n} H_{n}^{(1)}\left(\beta_{1} r\right) \sin n \theta
\end{aligned}
$$

and then the tangential stress $\sigma_{r \theta}$ has only the term about $\sin n \theta$, so at $\theta=0$ and $\theta=\pi$, and $\sigma_{r \theta}=0$ satisfies the free boundary condition of the straight boundary.

The total waves in the matrix material 1 can be obtained by the superposition principle

$$
\begin{aligned}
\phi_{1} & =\phi^{(i)+(r)}(r, \theta)+\phi_{1}^{(s)}(r, \theta) \\
& =\sum_{n=0}^{\infty} J_{n}\left(\alpha_{1} r\right)\left(a_{n} \cos n \theta+b_{n} \sin n \theta\right)+\sum_{n=1}^{\infty} A_{1 n} H_{n}^{(1)}\left(\alpha_{1} r\right) \sin n \theta,
\end{aligned}
$$

$$
\begin{aligned}
\psi_{1} & =\psi^{(r)}(r, \theta)+\psi_{1}^{(s)}(r, \theta) \\
& =\sum_{n=0}^{\infty} J_{n}\left(\beta_{1} r\right)\left(c_{n} \cos n \theta+d_{n} \sin n \theta\right)+\sum_{n=1}^{\infty} C_{1 n} H_{n}^{(1)}\left(\beta_{1} r\right) \sin n \theta
\end{aligned}
$$

The refracted waves on boundary $L_{1}$ are

$$
\begin{aligned}
& \phi^{(f)}(r, \theta)=\sum_{n=1}^{\infty} A_{2 n} J_{n}\left(\alpha_{2} r\right) \sin n \theta, \\
& \psi^{(f)}(r, \theta)=\sum_{n=1}^{\infty} C_{2 n} J_{n}\left(\beta_{2} r\right) \sin n \theta .
\end{aligned}
$$

The scattered waves generated by the refracted waves at interface $L_{1}$ are

$$
\begin{aligned}
& \phi_{2}^{(s)}(r, \theta)=\sum_{n=1}^{\infty} A_{3 n} H_{n}^{(2)}\left(\alpha_{2} r\right) \sin n \theta, \\
& \psi_{2}^{(s)}(r, \theta)=\sum_{n=1}^{\infty} C_{3 n} H_{n}^{(2)}\left(\beta_{2} r\right) \sin n \theta .
\end{aligned}
$$

The total waves in the shell 2 are obtained by the superposition principlee

$$
\begin{aligned}
\phi_{2}= & \phi^{(f)}(r, \theta)+\phi_{2}^{(s)}(r, \theta)=\sum_{n=1}^{\infty}\left[A_{2 n} J_{n}\left(\alpha_{2} r\right)+A_{3 n} H_{n}^{(2)}\right. \\
& \left.\cdot\left(\alpha_{2} r\right)\right] \sin n \theta, \\
\psi_{2}= & \psi^{(f)}(r, \theta)+\psi_{2}^{(s)}(r, \theta)=\sum_{n=1}^{\infty}\left[C_{2 n} J_{n}\left(\beta_{2} r\right)+C_{3 n} H_{n}^{(2)}\right. \\
& \left.\cdot\left(\beta_{2} r\right)\right] \sin n \theta,
\end{aligned}
$$

where $A_{1 n}, A_{2 n}, A_{3 n}$ and $C_{1 n}, C_{2 n}, C_{3 n}$ are unknown coefficients, determined by the boundary conditions at $L_{1}$ and $L_{2}$, $H_{n}^{(1)}(\cdot)$ is the first kind $n$-order Hankel function, $H_{n}^{(2)}(\cdot)$ is the second kind $n$-order Hankel function, $\alpha_{2}=\omega / c_{p 2}$ and $\beta_{2}=\omega / c_{s 2}$ are the wave numbers of the compression waves and shear waves in the shell, the compression waves velocity is $c_{p 2}=\sqrt{\lambda_{2}+2 \mu_{2} / \rho_{2}}$, and the shear waves velocity is $c_{s 2}=\sqrt{\mu_{2} / \rho_{2}}$.

2.3. Boundary Conditions. Substituting equations (32a) and (32b) into (18a)-(19c) for the displacements in the matrix, we get

$$
\begin{aligned}
& u_{r 1}=\frac{1}{r} \sum_{n=0}^{\infty}\left[\left(a_{n} M_{71}^{(1)}+d_{n} M_{72}^{(1)}+C_{1 n} M_{72}^{(3)}\right) \cos n \theta+\left(b_{n} M_{71}^{(1)}-c_{n} M_{72}^{(1)}+A_{1 n} M_{71}^{(3)}\right) \sin n \theta\right], \\
& u_{\theta 1}=\frac{1}{r} \sum_{n=0}^{\infty}\left[\left(d_{n} M_{82}^{(1)}+C_{1 n} M_{82}^{(3)}-a_{n} M_{81}^{(1)}\right) \sin n \theta+\left(b_{n} M_{81}^{(1)}+c_{n} M_{82}^{(1)}+A_{1 n} M_{81}^{(3)}\right) \cos n \theta\right] .
\end{aligned}
$$

The stresses in the matrix are

$$
\begin{aligned}
& \sigma_{r r 1}=\frac{2 \mu_{1}}{r^{2}} \sum_{n=0}^{\infty}\left[\left(a_{n} E_{11}^{(1)}+d_{n} E_{12}^{(1)}+C_{1 n} E_{12}^{(3)}\right) \cos n \theta+\left(b_{n} E_{11}^{(1)}+c_{n} E_{12}^{(1)}+A_{1 n} E_{11}^{(3)}\right) \sin n \theta\right], \\
& \sigma_{\theta \theta 1}=\frac{2 \mu_{1}}{r^{2}} \sum_{n=0}^{\infty}\left[\left(a_{n} E_{21}^{(1)}+d_{n} E_{22}^{(1)}+C_{1 n} E_{22}^{(3)}\right) \cos n \theta+\left(b_{n} E_{21}^{(1)}-c_{n} E_{22}^{(1)}+A_{1 n} E_{21}^{(3)}\right) \sin n \theta\right], \\
& \sigma_{r \theta 1}=\frac{2 \mu_{1}}{r^{2}} \sum_{n=0}^{\infty}\left[\left(d_{n} E_{42}^{(1)}+C_{1 n} E_{42}^{(3)}-a_{n} E_{41}^{(1)}\right) \sin n \theta+\left(b_{n} E_{41}^{(1)}+c_{n} E_{42}^{(1)}+A_{1 n} E_{41}^{(3)}\right) \cos n \theta\right] .
\end{aligned}
$$


Substituting equations (41a) and (41b) into equations (18a)-(19c), the displacements of the shell are

$$
\begin{aligned}
& u_{r 2}=\frac{1}{r} \sum_{n=0}^{\infty}\left[\left(A_{2 n} \overline{M_{71}^{(1)}}+A_{3 n} \overline{M_{71}^{(3)}}\right) \sin n \theta+\left(C_{2 n} \overline{M_{72}^{(1)}}+C_{3 n} \overline{M_{72}^{(3)}}\right) \cos n \theta\right], \\
& u_{\theta 2}=\frac{1}{r} \sum_{n=0}^{\infty}\left[\left(C_{2 n} \overline{M_{82}^{(1)}}+C_{3 n} \overline{M_{82}^{(3)}}\right) \sin n \theta+\left(A_{2 n} \overline{M_{81}^{(1)}}+A_{3 n} \overline{M_{81}^{(3)}}\right) \cos n \theta\right] .
\end{aligned}
$$

The stresses in the shell are

$$
\begin{aligned}
& \sigma_{r r 2}=\frac{2 \mu_{2}}{r^{2}} \sum_{n=0}^{\infty}\left[\left(A_{2 n} \overline{E_{11}^{(1)}}+A_{3 n} \overline{E_{11}^{(3)}}\right) \sin n \theta+\left(C_{2 n} \overline{E_{12}^{(1)}}+C_{3 n} \overline{E_{12}^{(3)}}\right) \cos n \theta\right], \\
& \sigma_{\theta \theta 2}=\frac{2 \mu_{2}}{r^{2}} \sum_{n=0}^{\infty}\left[\left(A_{2 n} \overline{E_{21}^{(1)}}+A_{3 n} \overline{E_{21}^{(3)}}\right) \sin n \theta+\left(C_{2 n} \overline{E_{22}^{(1)}}+C_{3 n} \overline{E_{22}^{(3)}}\right) \cos n \theta\right], \\
& \sigma_{r \theta 2}=\frac{2 \mu_{2}}{r^{2}} \sum_{n=0}^{\infty}\left[\left(A_{2 n} \overline{E_{41}^{(1)}}+A_{3 n} \overline{E_{41}^{(3)}}\right) \cos n \theta+\left(C_{2 n} \overline{E_{42}^{(1)}}+C_{3 n} \overline{E_{42}^{(3)}}\right) \sin n \theta\right] .
\end{aligned}
$$

Substituting equations (42a)-(45c) into the boundary conditional equations (10) and (13)-(16), using the orthogonality of $e^{i n \theta}$, multiplying $\sin n \theta$ at both ends of the question, and integrating $\theta$ in $[0, \pi]$, the specific form of the boundary conditional that needs to be satisfied by the abovementioned six unknown coefficients is

$$
\begin{aligned}
& \sum_{\substack{m=0 \\
n+m=\text { odd }}}^{\infty}\left(a_{m} M_{71}^{(1)}+d_{m} M_{72}^{(1)}+C_{1 m} M_{72}^{(3)}\right) s_{n m}+\frac{\pi}{2}\left(b_{n} M_{71}^{(1)}-c_{n} M_{72}^{(1)}+A_{1 n} M_{71}^{(3)}\right)-\frac{\pi}{2}\left(A_{2 n} \overline{M_{71}^{(1)}}+A_{3 n} \overline{M_{71}^{(3)}}\right) \\
& -\sum_{\substack{m=0 \\
n+m=\text { odd }}}^{\infty}\left(C_{2 m} \overline{M_{72}^{(1)}}+C_{3 m} \overline{M_{72}^{(3)}}\right) s_{n m}=0 \\
& \sum_{\substack{m=0 \\
n+m=\text { odd }}}^{\infty}\left(b_{m} M_{81}^{(1)}+c_{m} M_{82}^{(1)}+A_{1 m} M_{82}^{(3)}\right) s_{n m}+\frac{\pi}{2}\left(-a_{n} M_{81}^{(1)}+d_{n} M_{82}^{(1)}+C_{1 n} M_{82}^{(3)}\right)-\frac{\pi}{2}\left(C_{2 n} \overline{M_{82}^{(1)}}+C_{3 n} \overline{M_{82}^{(3)}}\right) \\
& -\sum_{\substack{m=0 \\
n+m=\text { odd }}}^{\infty}\left(A_{2 m} \overline{M_{81}^{(1)}}+A_{3 m} \overline{M_{81}^{(3)}}\right) s_{n m}=0 \\
& s_{1} \cdot \sum_{\substack{m=1 \\
n+m=\text { odd }}}^{\infty}\left[\left(\left(1-v_{2}\right) \overline{E_{22}^{(1)}}-v_{2} \overline{E_{12}^{(1)}}\right) C_{2 m}+\left(\left(1-v_{2}\right) \overline{E_{22}^{(3)}}-v_{2} \overline{E_{12}^{(3)}}\right) C_{3 m}\right] s_{n m}+s_{1} \cdot \frac{\pi}{2} \cdot\left[\left(\left(1-v_{2}\right) \overline{E_{21}^{(1)}}-v_{2} \overline{E_{11}^{(1)}}\right) A_{2 n}\right. \\
& \left.+\left(\left(1-v_{2}\right) \overline{E_{21}^{(3)}}-v_{2} \overline{E_{11}^{(3)}}\right) A_{3 n}\right]+\frac{\pi}{2} \cdot\left(A_{2 n} \overline{E_{11}^{(1)}}+A_{3 n} \overline{E_{11}^{(3)}}\right)+\sum_{\substack{m=1 \\
n+m=\text { odd }}}^{\infty}\left(C_{2 m} \overline{E_{12}^{(1)}}+C_{3 m} \overline{E_{12}^{(3)}}\right) s_{n m}=0,
\end{aligned}
$$




$$
\begin{aligned}
& s_{1} \cdot \sum_{\substack{m=0 \\
n+m=\text { odd }}}^{\infty} m \cdot\left[\left(\left(1-v_{2}\right) \overline{E_{21}^{(1)}}-v_{2} \overline{E_{11}^{(1)}}\right) A_{2 m}+\left(\left(1-v_{2}\right) \overline{E_{21}^{(3)}}-v_{2} \overline{E_{11}^{(3)}}\right) A_{3 m}\right] s_{n m}-s_{1} \cdot \frac{\pi}{2} \cdot n \cdot\left[\left(\left(1-v_{2}\right) \overline{E_{22}^{(1)}}-v_{2} \overline{E_{12}^{(1)}}\right) C_{2 n}\right. \\
& \left.+\left(\left(1-v_{2}\right) \overline{E_{22}^{(3)}}-v_{2} \overline{E_{12}^{(3)}}\right) C_{3 n}\right]-\frac{\pi}{2} \cdot\left(C_{2 n} \overline{E_{42}^{(1)}}+C_{3 n} \overline{E_{42}^{(3)}}\right)-\sum_{\substack{m=0 \\
n+m=\text { odd }}}^{\infty}\left(A_{2 m} \overline{E_{41}^{(1)}}+A_{3 m} \overline{E_{41}^{(3)}}\right) s_{n m}=0 \\
& s_{2} \cdot \sum_{\substack{m=0 \\
n+m=\text { odd }}}^{\infty}\left[\left(\left(1-v_{1}\right) E_{21}^{(1)}-v_{1} E_{11}^{(1)}\right) a_{m}+\left(\left(1-v_{1}\right) E_{22}^{(1)}-v_{1} E_{12}^{(1)}\right) d_{m}+\left(\left(1-v_{1}\right) E_{22}^{(3)}-v_{1} E_{12}^{(3)}\right) C_{1 m}\right] s_{n m}+s_{2} \cdot \frac{\pi}{2}\left[\left(\left(1-v_{1}\right) E_{21}^{(1)}\right.\right. \\
& \left.\left.-v_{1} E_{11}^{(1)}\right) b_{n}-\left(\left(1-v_{1}\right) E_{22}^{(1)}+v_{1} E_{12}^{(1)}\right) c_{n}+\left(\left(1-v_{1}\right) E_{21}^{(3)}-v_{1} E_{11}^{(3)}\right) A_{1 n}\right] \\
& -\frac{\pi}{2}\left(b_{n} E_{11}^{(1)}+c_{n} E_{12}^{(1)}+A_{1 n} E_{11}^{(3)}\right)-\sum_{\substack{m=0 \\
n+m=\text { odd }}}^{\infty}\left(a_{m} E_{11}^{(1)}+d_{m} E_{12}^{(1)}+C_{1 m} E_{12}^{(3)}\right) s_{n m}+\frac{\mu_{2}}{\mu_{1}} \sum_{\substack{m=0 \\
n+m=\text { odd }}}^{\infty}\left(C_{2 m} \overline{E_{12}^{(1)}}+C_{3 m} \overline{E_{12}^{(3)}}\right) s_{n m} \\
& +\frac{\pi}{2}\left(A_{2 n} \overline{E_{11}^{(1)}}+A_{3 n} \overline{E_{11}^{(3)}}\right) \frac{\mu_{2}}{\mu_{1}}=0 \\
& s_{2} \cdot \sum_{\substack{m=0 \\
n+m=\text { odd }}}^{\infty} m \cdot\left[\left(\left(1-v_{1}\right) E_{21}^{(1)}-v_{1} E_{11}^{(1)}\right) b_{m}+\left(\left(1-v_{1}\right) E_{22}^{(1)}-v_{1} E_{12}^{(1)}\right) c_{m}+\left(\left(1-v_{1}\right) E_{21}^{(3)}-v_{1} E_{11}^{(3)}\right) A_{1 m}\right] s_{n m}-s_{2} \cdot \frac{\pi}{2} \cdot n \\
& \cdot\left[\left(\left(1-v_{1}\right) E_{21}^{(1)}-v_{1} E_{11}^{(1)}\right) a_{n}+\left(\left(1-v_{1}\right) E_{22}^{(1)}-v_{1} E_{12}^{(1)}\right) d_{n}+\left(\left(1-v_{1}\right) E_{22}^{(3)}-v_{1} E_{12}^{(3)}\right) C_{1 n}\right] \\
& +\frac{\pi}{2}\left(-a_{n} E_{41}^{(1)}+d_{n} E_{42}^{(1)}+C_{1 n} E_{42}^{(3)}\right)+\sum_{\substack{m=0 \\
n+m=\text { odd }}}^{\infty}\left(b_{m} E_{41}^{(1)}+c_{m} E_{42}^{(1)}+A_{1 m} E_{41}^{(3)}\right) s_{n m}-\frac{\mu_{2}}{\mu_{1}} \sum_{\substack{m=0 \\
n+m=\text { odd }}}^{\infty}\left(A_{2 m} \overline{E_{41}^{(1)}}+A_{3 m} \overline{E_{41}^{(3)}}\right) s_{n m} \\
& -\frac{\pi}{2}\left(C_{2 n} \overline{E_{42}^{(1)}}+C_{3 n} \overline{E_{42}^{(3)}}\right) \frac{\mu_{2}}{\mu_{1}}=0
\end{aligned}
$$

where

$$
\begin{aligned}
& M_{71}^{(k)}=-n C_{n}\left(\alpha_{1} r\right)+\alpha_{1} r C_{n-1}\left(\alpha_{1} r\right), M_{72}^{(k)}=n C_{n}\left(\beta_{1} r\right) \\
& M_{81}^{(k)}=n C_{n}\left(\alpha_{1} r\right), M_{82}^{(k)}=n C_{n}\left(\beta_{1} r\right)-\beta_{1} r C_{n-1}\left(\beta_{1} r\right), \\
& E_{11}^{(k)}=\left(n^{2}+n-\frac{1}{2} \beta_{1}^{2} r^{2}\right) C_{n}\left(\alpha_{1} r\right)-\alpha_{1} r C_{n-1}\left(\alpha_{1} r\right), E_{12}^{(k)}=-\left(n^{2}+n\right) C_{n}\left(\beta_{1} r\right)+n \beta_{1} r C_{n-1}\left(\beta_{1} r\right), \\
& E_{21}^{(k)}=-\left(n^{2}+n+\frac{1}{2} \beta_{1}^{2} r^{2}-\alpha_{1}^{2} r^{2}\right) C_{n}\left(\alpha_{1} r\right)+\alpha_{1} r C_{n-1}\left(\alpha_{1} r\right), E_{22}^{(k)}=\left(n^{2}+n\right) C_{n}\left(\beta_{1} r\right)-n \beta_{1} r C_{n-1}\left(\beta_{1} r\right), \\
& E_{41}^{(k)}=-\left(n^{2}+n\right) C_{n}\left(\alpha_{1} r\right)+n \alpha_{1} r C_{n-1}\left(\alpha_{1} r\right), E_{42}^{(k)}=-\left(n^{2}+n-\frac{1}{2} \beta_{1}^{2} r^{2}\right) C_{n}\left(\beta_{1} r\right)-\beta_{1} r C_{n-1}\left(\beta_{1} r\right),
\end{aligned}
$$




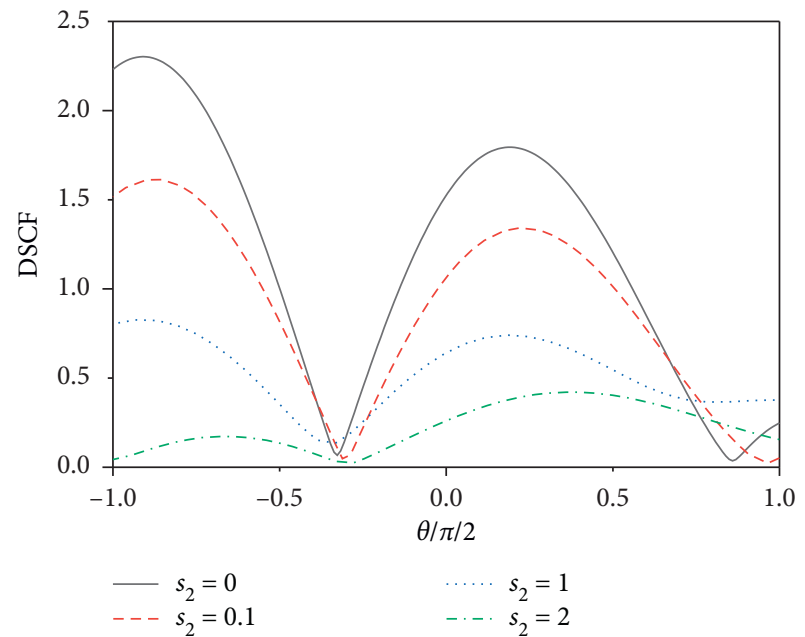

FIGURE 2: Distribution of DSCF along the interface $\left(r_{1}=a\right)$ with various values of $s_{2}$ under $\alpha_{1} a=0.2, \quad \eta=1.1, \quad \mu_{2} / \mu_{1}=0.2$, $\alpha_{2} / \alpha_{1}=1.5$.

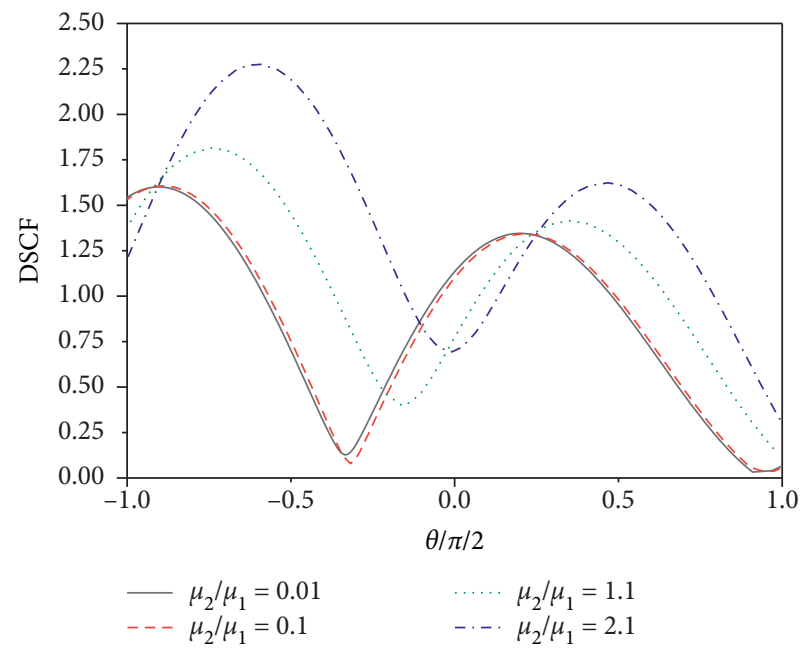

FIGURE 3: Distribution of DSCF along the interface $\left(r_{1}=a\right)$ with various values of $\mu_{2} / \mu_{1}$ under $\alpha_{1} a=0.2, s_{2}=0.1, \quad \eta=1.1$, $\alpha_{2} / \alpha_{1}=1.5$.

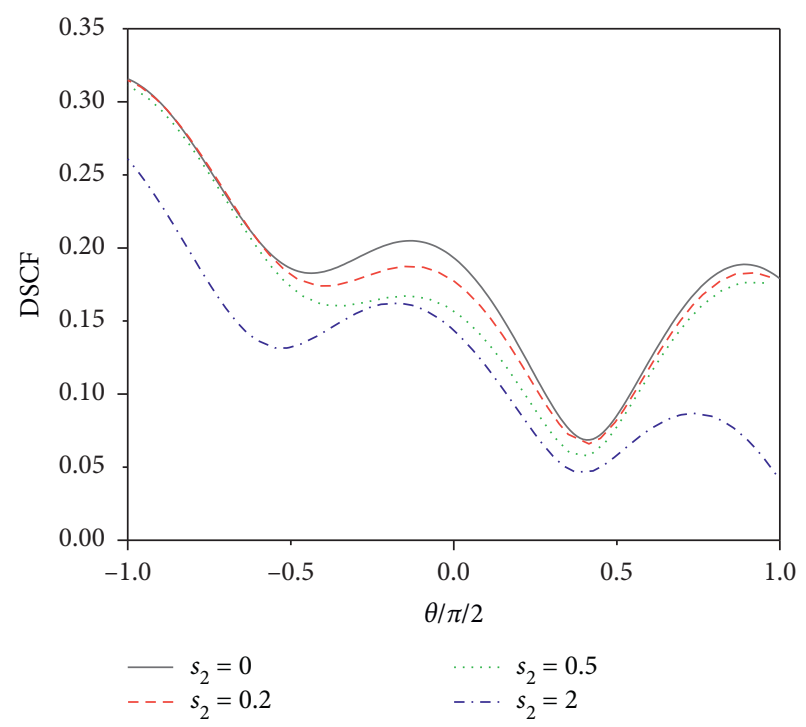

FIgURE 4: Distribution of DSCF along the interface $\left(r_{1}=a\right)$ with various values of $s_{2}$ under $\alpha_{1} a=\pi, \eta=1.1, \mu_{2} / \mu_{1}=0.2, \alpha_{2} / \alpha_{1}=1.5$.

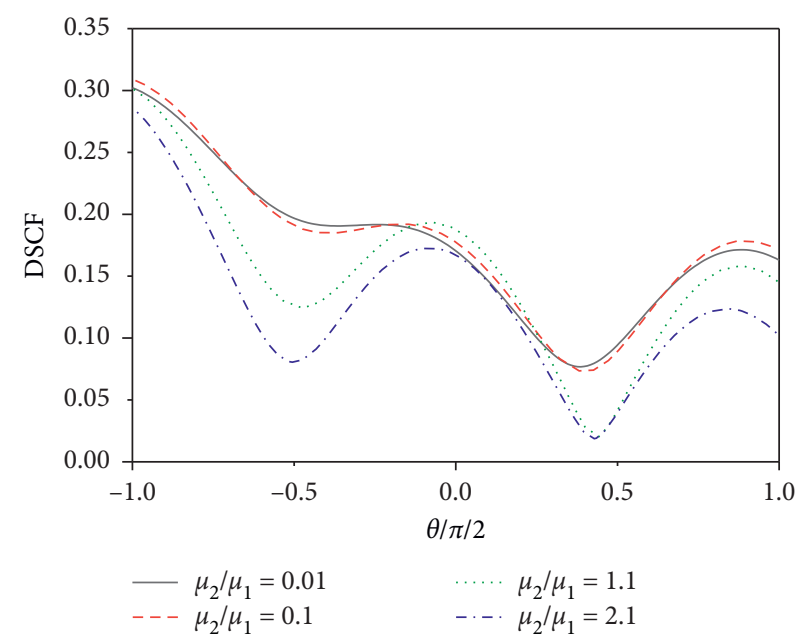

FIgURE 5: Distribution of DSCF along the interface $\left(r_{1}=a\right)$ with various values of $\mu_{2} / \mu_{1}$ under $\alpha_{1} a=\pi, s_{2}=0.1, \eta=1.1, \alpha_{2} / \alpha_{1}=1.5$.

$$
\begin{aligned}
C_{n}(\cdot) & =H_{n}^{(1)}(\cdot), \\
\overline{M_{71}^{(k)}} & =-n C_{n}\left(\alpha_{2} r\right)+\alpha_{2} r C_{n-1}\left(\alpha_{2} r\right), \overline{M_{72}^{(k)}}=n C_{n}\left(\beta_{2} r\right), \\
\overline{M_{81}^{(k)}} & =n C_{n}\left(\alpha_{2} r\right), \overline{M_{82}^{(k)}}=n C_{n}\left(\beta_{2} r\right)-\beta_{2} r C_{n-1}\left(\beta_{2} r\right), \\
\overline{E_{11}^{(k)}} & =\left(n^{2}+n-\frac{1}{2} \beta_{2}^{2} r^{2}\right) C_{n}\left(\alpha_{2} r\right)-\alpha_{2} r C_{n-1}\left(\alpha_{2} r\right), \overline{E_{12}^{(k)}}=-\left(n^{2}+n\right) C_{n}\left(\beta_{2} r\right)+n \beta_{2} r C_{n-1}\left(\beta_{2} r\right), \\
E_{21}^{(k)} & =-\left(n^{2}+n+\frac{1}{2} \beta_{2}^{2} r^{2}-\alpha_{2}^{2} r^{2}\right) C_{n}\left(\alpha_{2} r\right)+\alpha_{2} r C_{n-1}\left(\alpha_{2} r\right), \overline{E_{22}^{(k)}}=\left(n^{2}+n\right) C_{n}\left(\beta_{2} r\right)-n \beta_{2} r C_{n-1}\left(\beta_{2} r\right), \\
\overline{E_{41}^{(k)}} & =-\left(n^{2}+n\right) C_{n}\left(\alpha_{2} r\right)+n \alpha_{2} r C_{n-1}\left(\alpha_{2} r\right), \overline{E_{42}^{(k)}}=-\left(n^{2}+n-\frac{1}{2} \beta_{2}^{2} r^{2}\right) C_{n}\left(\beta_{2} r\right)-\beta_{2} r C_{n-1}\left(\beta_{2} r\right), \\
k & =1, C_{n}(\cdot)=J_{n}(\cdot), k=3, C_{n}(\cdot)=H_{n}^{(2)}(\cdot) .
\end{aligned}
$$




\section{Results and Discussion}

The following is an analysis of the variation of the DSCF under the different conditions. The DSCF at the interface of a semicylindrical core-shell structure is usually defined as

$$
\mathrm{DSCF}=\left|\frac{\sigma_{\theta \theta 1}}{\sigma_{0}}\right|,
$$

where $\sigma_{\theta \theta 1}$ is the bulk stress of the matrix material at interface $r=a$, and $\sigma_{0}=-\mu_{1} \beta_{1}^{2} \phi_{0}$ is the stress intensity in the propagation direction of $\mathrm{P}$ waves.

It is seen that when the surface/interface effect is taken into account, the dynamic stress depends not only on the wave number and Poisson's ratio but also on the surface elasticity parameter $s$. After considering the surface effect and the semicylindrical core-shell structure model, DSCF is related not only to the wave number of the incident waves but also to factors such as surface parameters and angle of incidence. The following is a study of the variation of the dynamic stress concentration factor by taking different material constants, and we keep $\nu_{1}=v_{2}=0.25$ and $\eta=1.1$ in the calculations.

3.1. The Effects of a Low-Frequency Incident Wave on DSCF, That as $\alpha_{1} a=0.2$. In this case, the wavelength $\lambda=40 \pi a$ of the incident waves is much large than the radius of the shell and the core layer. Figures 2 and 3 show the distributions of DSCF near the surface $(r=a)$ of the shell for different values of $s_{2}$ and $\mu_{2} / \mu_{1}$. It can be seen from Figure 2 that the effect factor $s_{2}$ of surface/interface has a significant influence on the DSCF on the surface of the shell. As $s_{2}$ increases, the DSCF decreases significantly around $\theta=-\pi / 2$ and $\theta=\pi / 8$, with minor change at $\theta=\pi / 5$ and $\theta=3 \pi / 8$.

To detect the overall performance impact, Figure 3 shows the DSCF near the semicylindrical core-shell structure for various ratios of shear modulus $\mu_{2} / \mu_{1}$, when the shear modulus increased, the DSCF significantly increased around $\theta= \pm \pi / 4$, and slightly decreased around $\theta=0$. For softer inclusions $\left(\mu_{2} / \mu_{1}<1\right)$, the peak of DSCF appears near $\theta=0$ and $\theta=\pi / 8$, and for harder inclusions $\left(\mu_{2} / \mu_{1}>1\right)$, the maximum value of DSCF appears at $\theta=-\pi / 4$.

3.2. The Effects of a High-Frequency Incident Wave on DSCF, That as $\alpha_{1} a=3.14$. In this case, the wavelength $\lambda=2$ of the incident waves equals to the diameter of the core-shell inclusions. Figures 4 and 5 show the distribution of DSCF near the surface $(r=a)$ of the shell for different values of $s_{2}$ and $\mu_{2} / \mu_{1}$, and it was generally lower than that of a low frequency incident waves. Figure 4 shows that the effect factor $s_{2}$ of surface/interface has a significant influence on the DSCF on the surface of the shell. As $s_{2}$ increases, the DSCF decreases significantly around $\theta=\pi / 2$, with little change at $\theta=\pi / 5$. As can be seen from Figure 4, when $s_{2}=2$, the amplitude of DSCF drops faster than $s_{2}<2$.

In Figure 5 when the $\mu_{2} / \mu_{1}$ increased, the DSCF decreased significantly around $\theta= \pm \pi / 4$, with minor change at $\theta=\pi / 8$. For softer inclusions $\left(\mu_{2} / \mu_{1}<1\right)$, the amplitude of DSCF does not change substantially with the value of $\mu_{2} / \mu_{1}$, and for harder inclusions $\left(\mu_{2} / \mu_{1}>1\right)$, the magnitude of DSCF varies significantly with the value of $\mu_{2} / \mu_{1}$.

\section{Conclusions}

In this paper, the wave function expansion method and the conversion formula of the sine function and cosine function are used to theoretically study the scattering characteristics of plane compression waves of a semicylindrical core-shell structure, solutions for the elastic fields induced by P-waves near the surface of the semicylindrical core-shell structure is obtained, and the effects of interface properties on the dynamic stress concentration factors near the surface of the semicylindrical core-shell nanostructures are discussed in detail. The results show that as the shell radius is reduced to the nanometer level, the incident frequency of the incident wave has a significant effect on DSCF. Hard inclusions are more easily affected than soft inclusions, and the dynamic stress concentration factor changes with the interface effect, significantly.

\section{Data Availability}

The data used to support the findings of this study are included within the article.

\section{Conflicts of Interest}

The authors declare that there are no conflicts of interest regarding the publication of this paper.

\section{Acknowledgments}

The support from the National Natural Science Foundation (Grant nos. 11362009 and 11862014) are acknowledged.

\section{References}

[1] S. Iijima, "Helical microtubules of graphitic carbon," Nature, vol. 354, no. 6348, pp. 56-58, 1991.

[2] V. Twersky, "Multiple scattering of radiation by an arbitrary planar configuration of parallel cylinders and by two parallel cylinders," Journal of Applied Physics, vol. 23, no. 4, pp. 402-414, 1952.

[3] J. G. Fikioris and P. C. Waterman, "Multiple scattering of waves. II. "Hole corrections" in the scalar case," Journal of Mathematical Physics, vol. 5, no. 10, pp. 1413-1420, 1964.

[4] P. C. Waterman and R. Truell, "Multiple scattering of waves," Journal of Mathematical Physics, vol. 2, no. 4, pp. 512-537, 1961.

[5] V. Twersky, "Multiple scattering of sound by a periodic line of obstacles," The Journal of the Acoustical Society of America, vol. 53, no. 1, pp. 96-112, 1973.

[6] V. K. Varadan, V. V. Varadan, and Y. H. Pao, "Multiple scattering of elastic waves by cylinders of arbitrary cross section. I. SH waves," The Journal of the Acoustical Society of America, vol. 63, no. 5, pp. 1310-1319, 1978.

[7] P. Sheng, Introduction to Wave Scattering, Localization and Mesoscopic Phenomena, Academic Press, Cambridge, MA, USA, 2006.

[8] L. Tsang and J. A. Kong, Scattering of Electromagnetic Waves: Advanced Topics, Wiley, Hoboken, NJ, USA, 2001. 
[9] Y. H. Pao and C. C. Mow, "Dynamic stress concentration in an elastic plate with rigid circular inclusion," in Proceedings of the 4th National Congress of Applied Mechanics, pp. 335-340, Berkeley, CA, USA, June 1962.

[10] C. C. Mow and L. J. Mente, "Dynamic stresses and displacements around cylindrical discontinuities due to plane harmonic shear waves," Journal of Applied Mechanics, vol. 30, no. 4 , pp. 598-604, 1963.

[11] J. A. Roumeliotis and J. G. Fikioris, "Cutoff wavenumbers and the field of surface wave modes of an eccentric circular goubau waveguide," Journal of the Franklin Institute, vol. 309, no. 5, pp. 309-325, 1980.

[12] M. D. Trifunac, "Scattering of plane SH waves by a semicylindrical canyon," Earthquake Engineering \& Structural Dynamics, vol. 1, no. 3, pp. 267-281, 1972.

[13] W. Weber, B. W. Zastrau, and W. Bernd, "Non-plane wave scattering from a single eccentric circular inclusion- Part 1:SH waves," Journal of Theoretical and Applied Mechanics, vol. 49, pp. 1183-1201, 2011.

[14] J.-w. Liang, L.-j. Yan, and V. W. Lee, "Scattering of plane P waves by circular-arc layered alluvial valleys: an analytical solution," Acta Seismologica Sinica, vol. 14, no. 2, pp. 176-195, 2001.

[15] Q. Bo, C. Jiu-Jiu, and J.-C. Cheng, "Local resonant characteristics of a layered cylinder embedded in the elastic medium," Chinese Physics, vol. 14, no. 12, pp. 2522-2528, 2005.

[16] D. G. Li, X. P. Gao, and Z. Ying, "An analytic solution for the diffraction of plane $P$ waves by a cylindrical inclusion in half space," Advanced Materials Research, vol. 255-260, pp. 2520-2525, 2011.

[17] Y. Shindo and N. Niwa, "Scattering of antiplane shear waves in a fiber-reinforced composite medium with interfacial layers," Acta Mechanica, vol. 117, no. 1-4, pp. 181-190, 1996.

[18] V. W. Lee and W.-Y. Liu, "Two-dimensional scattering and diffraction of $\mathrm{P}$ - and $\mathrm{SV}$-waves around a semi-circular canyon in an elastic half-space: an analytic solution via a stress-free wave function," Soil Dynamics and Earthquake Engineering, vol. 63, pp. 110-119, 2014.

[19] Y. Xu, Y. Li, L. Cao, Z. Yang, and X. Zhou, "Steering of SH wave propagation in electrorheological elastomer with a structured meta-slab by tunable phase discontinuities," AIP Advance, vol. 7, no. 9, Article ID 095114, 2017.

[20] L. Cao, Z. Yang, and Y. Xu, "Steering elastic SH waves in an anomalous way by metasurface," Journal of Sound and Vibration, vol. 418, pp. 1-14, 2018.

[21] G. F. Wang, T. J. Wang, and X. Q. Feng, "Surface effects on the diffraction of plane compressional waves by a nano-sized circular hole," Applied Physics Letters, vol. 89, no. 23, pp. 1923-1930, 2006.

[22] G. F. Wang, "Multiple diffraction of plane compressional waves by two circular cylindrical holes with surface effects," Journal of Applied Physics, vol. 105, pp. 1-6, 2009.

[23] Z. Y. Ou and D. W. Lee, "Effects of interface energy on scattering of plane elastic wave by a nano-sized coated fiber," Journal of Sound and Vibration, vol. 331, no. 25, pp. 5623$5643,2012$.

[24] Y. Ru, G. F. Wang, and T. J. Wang, "Diffractions of elastic waves and stress concentration near a cylindrical nano-inclusion incorporating surface effect," Journal of Vibration and Acoustics, vol. 131, no. 6, pp. 1-7, 2009.

[25] C. X. Kan, W. P. Cai, and L. D. Zhang, "Core-shell structure composite nanoparticles,” Physics, vol. 3, pp. 146-150, 2002.

[26] L. Heng, D. Tian, L. Chen et al., "Local photoelectric conversion properties of titanyl-phthalocyanine (TiOPc) coated aligned ZnO nanorods," Chemical Communications, vol. 46, no. 7, pp. 1162-1164, 2010.

[27] H. Ren, Q. Huang, and X. Chen, "Existence of evanescent electromagnetic waves resulting from seismoelectric conversion at a solid-porous interface," Geophysical Journal International, vol. 204, no. 1, pp. 147-166, 2016.

[28] W. U. Huynh, "Hybrid nanorod-polymer solar cells," Science, vol. 295, no. 5564, pp. 2425-2427, 2002.

[29] S. S. Kanmani and K. Ramachandran, "Synthesis and characterization of $\mathrm{TiO}_{2} / \mathrm{ZnO}$ core/shell nanomaterials for solar cell applications," Renewable Energy, vol. 43, pp. 149-156, 2012.

[30] M. F. Kircher, U. Mahmood, R. S. King et al., "A multimodal nanoparticle for preoperative magnetic resonance imaging and intraoperative optical brain tumor delineation," Cancer Research, vol. 63, no. 23, pp. 8122-8125, 2004.

[31] L. M. Tang, T. J. Huang, and H. Song, "Research progress of core-shell nanocomposites," Materials Reports, vol. 30, no. S1, p. 40-42, 2016.

[32] R. G. Chaudhuri and S. Paria, "Core/shell nanoparticles: classes, properties, synthesis mechanisms, characterization, and applications," Chemical Reviews, vol. 112, no. 4, pp. 2373-2433, 2012.

[33] S. K. Asl, M. K. Rad, and S. K. Sadrnezhaad, "Hydrothermal preparation of $\mathrm{TiO}_{2}-\mathrm{ZnO}$ nano core-shell structure with quantum size effect," in Proceedings of the AIP Conference, vol. 1400, pp. 425-428, Geneva, Switzerland, 2011.

[34] Z. Libor and Q. Zhang, "The synthesis of nickel nanoparticles with controlled morphology and $\mathrm{SiO}_{2} / \mathrm{Ni}$ core-shell structures," Materials Chemistry and Physics, vol. 114, no. 2-3, pp. 902-907, 2009. 